\title{
Decompressive surgery in malignant dural sinus thrombosis: report of 3 cases and review of the literature
}

\author{
Luigi A. Lanterna, M.D., Ph.D., ${ }^{1}$ Paolo Gritti, M.D., ${ }^{2}$ Ornella Manara, M.D., ${ }^{3}$ \\ Gianluca Grimod, M.D., ${ }^{1}$ Gianmario Bortolotti, M.D., ${ }^{2}$ and Francesco Biroli, M.D., 1 \\ Departments of ${ }^{1}$ Neuroscience and Surgery of the Nervous System, ${ }^{2}$ Neuroanesthesiology, and \\ ${ }^{3}$ Neuroradiology, Ospedali Riuniti, Bergamo, Italy
}

\begin{abstract}
Cerebral venous and dural sinus thrombosis (CVDST) is a rare cause of stroke in young and middle-aged adults. When the clinical course is complicated by uncontrollable intracranial hypertension and brainstem compression due to edema or cerebral hemorrhage, the prognosis is poor. The authors evaluated the therapeutic role of surgical decompression in patients with clinical signs of impending herniation. Cerebral venous and dural sinus thrombosis complicated by impending brain herniation a very rare, life-threatening but potentially treatable clinical condition.

Three patients with pupillary signs of transtentorial herniation due to brain edema and hemorrhage caused by CVDST (superior sagittal sinus in 1 patient and transverse and sigmoid sinus in 2 patients) were treated surgically. The intervention consisted of clot removal, infarcted tissue resection, and frontotemporoparietooccipital craniectomy with duraplasty. According to the Glasgow Outcome Scale, 2 patients were classified as having good recovery and 1 , moderate disability. The results of neuropsychological assessment were normal in 2 patients and demonstrated a partial neuropsychological deficit (neglect) in the other.

Surgery may be indicated in selected patients with CVDST whose condition is deteriorating because of intractable intracranial hypertension and impending brain herniation. (DOI: 10.3171/2009.3.FOCUSO910)
\end{abstract}

KEY WoRds $\bullet \quad$ cerebral venous thrombosis $\bullet \quad$ craniectomy
intracranial pressure $\bullet \quad$ dural sinus $\bullet \quad$ hemorrhagic infarct

$\mathrm{C}$ EREBRAL vein and dural sinus thrombosis predominates in young and middle-aged adults. Despite improvements in diagnosis and treatment, more than $15 \%$ of patients remain permanently disabled or die. ${ }^{7,9}$ Recently, the International Study on Cerebral Vein and Dural Sinus Thrombosis (ISCVT) has shown that the most common mechanism of acute death is transtentorial herniation due to hemorrhagic lesions or diffuse brain edema. ${ }^{1,2}$ In these circumstances, decompressive surgery (DS) may be the treatment to choose as it promptly creates space for swelling cerebral tissue and may also improve perfusion of collateral leptomeningeal vessels.

The ISCVT suggested the importance of investigating DS with randomized trials. ${ }^{7}$ However, the rarity of the pathology (5 cases per million), ${ }^{1}$ and the fact that only a minority of patients with CVDST suffer from impending brain herniation necessitating DS, means that such a study is unlikely to succeed, even with long-term international collaborative efforts.

We report the cases of 3 patients with pupillary signs of impending brain herniation secondary to CVDST who underwent DS on an emergency basis, and we discuss the

\footnotetext{
Abbreviations used in this paper: CVDST $=$ cerebral venous and dural sinus thrombosis; GOS = Glasgow Outcome Scale; ICP = intracranial pressure; ISCVT = International Study on Cerebral Vein and Dural Sinus Thrombosis.
}

rationale and the clinical results of the procedure in relation to the physiopathology of CVDST.

\section{Methods}

Source of Data

We reviewed a prospectively collected database of the patients admitted for cerebrovascular pathological conditions to the Neurosurgical Intensive Care Unit of the Ospedali Riuniti of Bergamo from January 2006 to November 2008. A patient was eligible if a diagnosis of CVDST was confirmed by angiography, CT venography, MR imaging, or autopsy. Exclusion criteria were: age $<18$ years, cerebral lesions other than those related to CVDST, and posttraumatic, postinfective, or iatrogenic CVDSTs.

\section{Causal Treatment (Anticoagulation Therapy)}

Causal treatment was started at the diagnosis of CVDST and consisted of low-molecular-weight heparin (Nadroparin) in a dose of approximately 180 anti-factor Xa units $/ \mathrm{kg}$ per 24 hours or dose-adjusted intravenous heparin with an at least doubled activated partial thromboplastin time. Cerebral hemorrhage was not a contraindication against causal treatment. Patients in critical condition who could become potential surgical candidates were preferentially treated with unfractionated heparin because it has the advantage of being promptly antago- 


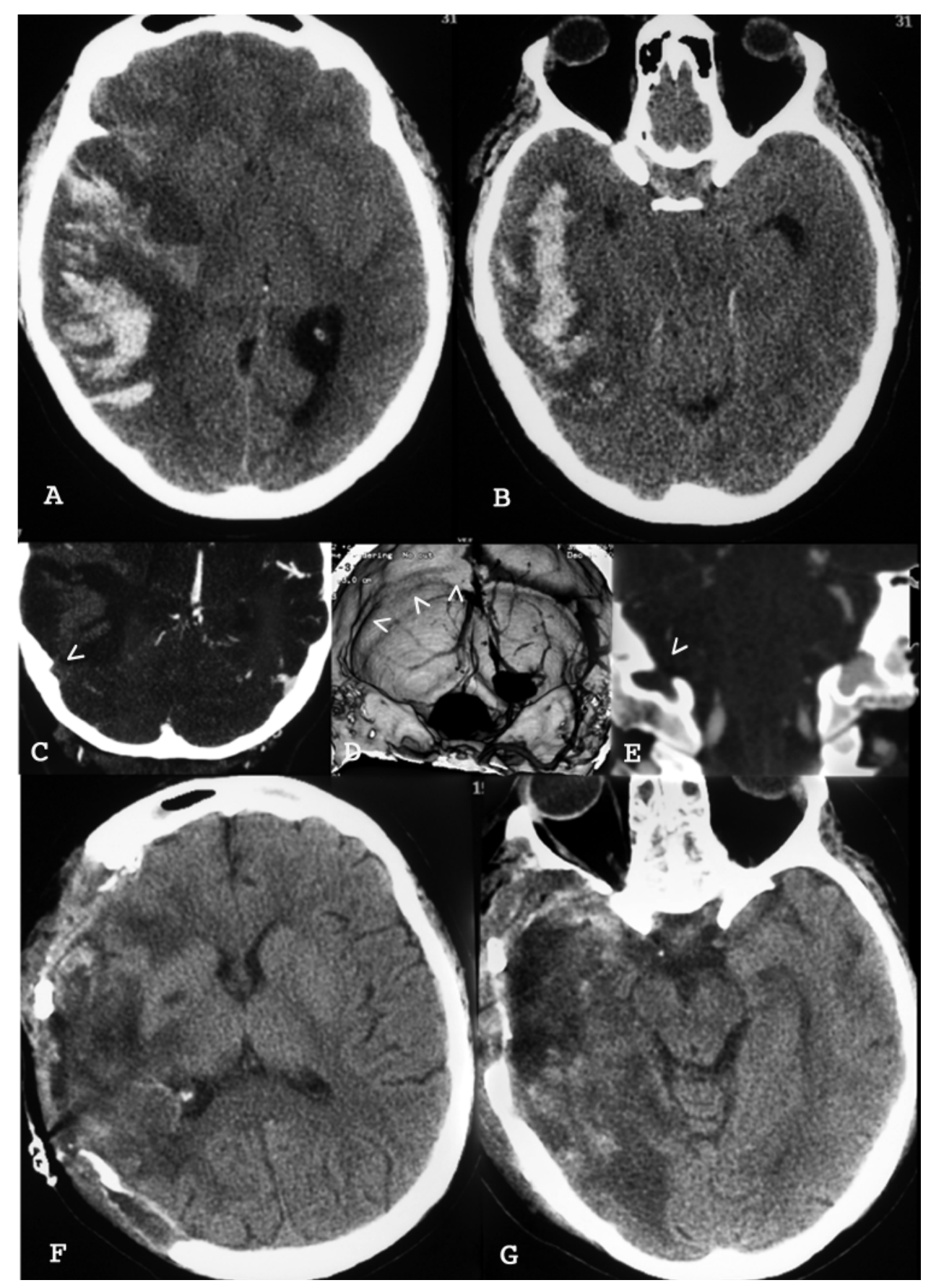

FIG. 1. Case 1. A and B: Preoperative CT revealing a hemorrhagic infarction of the right temporal lobe with midline shift and transtentorial herniation brainstem compression. $\quad \mathrm{C}$ and D: Preoperative CT angiograms demonstrating the non-opacification of the transverse sinus on the right side. The arrowheads indicate the transverse sinus sulcus. E: Preoperative CT angiogram in frontal projection showing the non-opacification of the jugular bulb on the right side; the jugular foramens are symmetrical. The arrowheads indicate the jugular foramen. F and G: Postoperative CT obtained after the second operation.

nized. In the other patients, low-molecular-weight heparins were preferred because they appear to be as effective as unfractionated heparin, they cause fewer hemorrhagic complications, and they can be given at fixed doses without constant laboratory monitoring. ${ }^{4,14}$ The anticoagulation therapy was never interrupted. It was maintained with a half dosage for the first 24-72 hours after surgery (during the period of major postoperative hemorrhage risk). ${ }^{8}$ The temporary reduction of dosage is in accordance with the guidelines of both the European Federation of Neurological Societies and the ISCVT treatment protocol, which suggest the temporary interruption of anticoagulation therapy in the event of invasive procedures.,

\section{Decompressive Surgery}

Indications for DS included: progressive neurologi- 
cal deterioration unresponsive to causal treatment and conventional intensive care (osmotherapy, moderate hyperventilation with a $\mathrm{pCO}_{2}$ of $30-35 \mathrm{~mm} \mathrm{Hg}$ and hypothermia with a target temperature of $33-35^{\circ} \mathrm{C}$ ) and $\mathrm{CT}$ signs of space-occupying lesions with midline shift or obliteration of the basal cisterns and pupillary signs of transtentorial herniation. Written consent for surgery was obtained from the patient or his or her next of kin. Decompressive surgery was defined in terms of external and internal decompression. External decompression was defined as a hemicraniectomy with duraplasty (diameter $\geq$ $12 \mathrm{~cm}$ ), and internal decompression consisted of resecting the infarcted brain tissue and draining cerebral hematomas. External decompression was primarily indicated in the event of diffuse hematomas infiltrating edematous, potentially still viable, eloquent brain, whereas internal decompression was primarily indicated in the event of localized hematomas in infarcted noneloquent brain parenchyma. Internal decompression was performed under microscopic magnification. During the operation the patients were rehydrated with isotonic crystalloids with care taken to maintain euvolemia and a serum osmolality of $300 \mathrm{mOsm} / \mathrm{L}$. The target hematocrit was kept at about $30 \%$ to reduce blood viscosity. ${ }^{6}$ Colloids and inotropes were used to keep the mean arterial blood pressure level above $90 \mathrm{~mm} \mathrm{Hg}$. Postoperative ICP monitoring was performed in all patients who underwent DS. In the event of postoperative ICP elevation a CT scan was performed and the indications for repeated surgery were ruled out. Those patients who were not candidates for repeated surgery were eligible for barbiturate coma with the aim of achieving electroencephalographic burst suppression.

\section{Case Reports}

\section{Case 1}

History, Examination, and Diagnosis. This 32-yearold right-handed woman was transferred to our institution from another hospital where she had presented in deep coma after a 12-hour history of headache. At examination, the patient was comatose with no motor response to pain and bilaterally fixed and maximally dilated pupils. A CT scan of the brain revealed a hemorrhagic infarct of the temporal lobe on the right side, brain edema, and brainstem compression (Figs. 1A and B). A CT angiography study demonstrated nonvisualization of the transverse and sigmoid sinuses on the right side (Fig. 1C, D, and $\mathrm{E}$ ). Intravenous mannitol and hyperventilation were ineffective.

Operation and Postoperative Course. Emergency decompressive surgery was planned, and a large frontotemporoparietal hemicraniectomy was performed. The sylvian veins were congested and the vein of Labbé appeared thrombotic. An intraparenchymal superficial clot was removed, while the infarcted cerebral tissue was only partially resected with the rationale of sparing edematous, potentially still viable brain. At the end of the operation the brain appeared slack, well perfused, and pulsating, and the ICP was $5 \mathrm{~mm} \mathrm{Hg}$. The postoperative CT scan showed complete clot removal and reduction of the midline shift. On Day 3, the ICP increased up to $25 \mathrm{~mm} \mathrm{Hg}$ and a CT scan revealed an increased mass effect due to edema and bleeding of the infarcted temporal lobe. Hypothermia and barbiturate coma were effective in lowering the ICP to 15-18 $\mathrm{mm} \mathrm{Hg}$ until Day 5, when the ICP rose to $30 \mathrm{~mm} \mathrm{Hg}$ and the right pupil became maximally dilated and unreactive to light. The patient was returned to the operating room, where the craniotomy was enlarged at the site of the prior parietooccipital craniotomy and the temporal squama was extensively drilled up to the cranial base. The bone bleeding was abnormally intense, suggesting compensatory venous drainage through the emissary veins. The theoretical hypothesis that craniotomy may affect the osseous compensatory circuits was not a contraindication to DS because of the multiplicity of the emissary veins and the wide distribution of the diploic venous network. ${ }^{12}$ The drilling was extended from the superior orbital fissure to the posterior root of the zygomatic arch with the aim of maximizing the decompression and obtaining an unobstructed view of the temporal lobe from the lateral, basal, and medial surfaces. This exposure was considered necessary to control possible bleeding from the congested and hypertrophic sylvian, temporobasal, and polar veins that were compensating for the thrombosed vein of Labbé. The dura mater was opened. The brain appeared diffusely swollen and the sylvian veins as well as the anterior temporal veins appeared red and congested. The infarcted tissue of the second and third temporal gyri was resected with extreme care taken to preserve all the veins and spare the mesial temporal structures that were not involved in the infarction. Once the infarcted tissue had been resected, the temporal and sylvian veins normalized and the brain swelling rapidly reduced. Intraoperatively, the ambient cistern and the brainstem appeared decompressed from the uncus. The postoperative CT scan showed complete clot removal, decompression of the ambient cistern, and absence of midline shift (Fig. 1F and G).

Outcome. At the 6-month follow-up examination the patient's GOS score indicated moderate disability because of partial neglect and a mild monoparesis of the left arm. She suffered from seizures, which were controlled with phenobarbital.

Case 2

History, Examination, and Diagnosis. This 51-yearold left-handed man was admitted to another hospital because of headache, confusion, and mild right-sided hemiparesis. A CT scan showed an intracerebral hematoma and brain edema of the left parietal lobe with midline shift. He was transferred to our institution, where digital subtraction angiography demonstrated slow circulation, stagnation of the contrast medium in the frontoparietal ascending veins, and no opacification of the superior sagittal sinus, suggestive of thrombosis (Fig. 2B and C). On Day 4, the right-sided hemiparesis worsened and a CT scan revealed the enlargement of the hematoma and worsening of the edema and the mass effect (Fig. 2A). Osmotics were ineffective and the following day he became 


\section{A. Lanterna et al.}
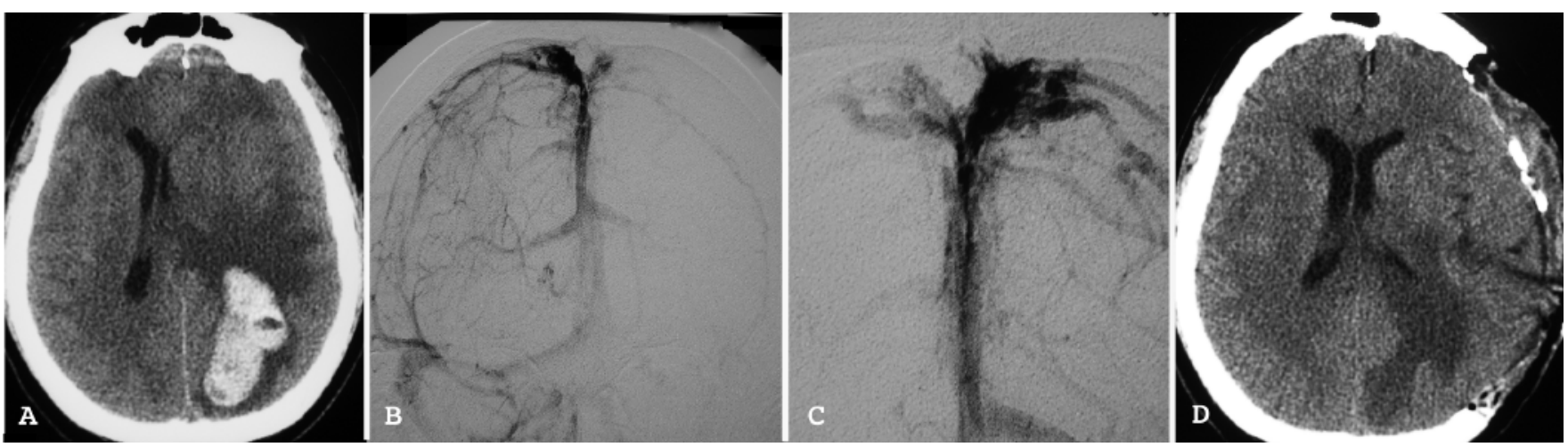

FIG. 2. Case 2. A: Preoperative CT revealing a hemorrhagic infarction of the left parietal lobe with brain swelling and midline shift. $\quad B$ and $C$ : Late-phase right $(B)$ and left $(C)$ internal carotid artery digital subtraction angiograms in frontal projection, demonstrating stagnation of the contrast medium in the frontoparietal ascending veins and no opacification of the superior sagittal sinus. D: Postoperative CT scan of the brain.

comatose with an abnormal flexion response to pain and a dilated pupil on the left side.

Operation and Postoperative Course. A large frontotemporoparietooccipital hemicraniectomy was performed. The bone bleeding was abnormally intense. The dura mater was opened. The brain appeared swollen and the cortical veins draining into the sagittal sinus were congested and red. The clot was evacuated together with the bleeding, congested, infarcted tissue surrounding the hematoma. The ICP remained $\leq 15 \mathrm{~mm} \mathrm{Hg}$. On Day 7, sedation was discontinued and the patient began to respond to commands. The postoperative CT scan showed complete clot removal and reduction of the midline shift (Fig. 2D).

Outcome. After 3 months the bone flap was repositioned. The patient returned to his normal daily life; his GOS score indicated good recovery and the neuropsychological evaluation was normal. He suffered from seizures, which were controlled with carbamazepine.

Case 3

History, Examination, and Diagnosis. This 39-yearold left-handed man was admitted to the emergency department because of headache, vomiting and a reduced level of consciousness (GCS score 12). A CT scan showed a hemorrhagic infarct of the temporal lobe on the right side, brain swelling, and compression of the ambient cistern (Fig. 3A). An MR imaging study demonstrated the absence of a flow signal into the transverse and sigmoid sinuses on the right side (Fig. 3B). Diffusion-weighted images revealed predominant cytotoxic edema. During the next 3 hours the patient's condition deteriorated further and, on reevalution, he was comatose (GCS score 8) with dilation of the pupil on the right side. After resuscitation with tracheal intubation, artificial hyperventilation, and mannitol, the pupillary diameter normalized.

Operation and Postoperative Course. Emergency decompressive surgery was planned and a large frontotemporoparietal hemicraniectomy, with drilling of the temporal bone, was performed. The temporal lobe was swollen, pale, and nonpulsating. The vein of Labbé was thrombosed while the sylvian and anterior temporal veins were prominent and congested. The hemorrhagic infarct was resected (middle and inferior temporal gyrus) with extreme care taken to preserve all the veins and to spare the mesial temporal structures. Postoperative CT showed complete clot removal and reduction of the temporal lobe swelling (Fig. 3C).
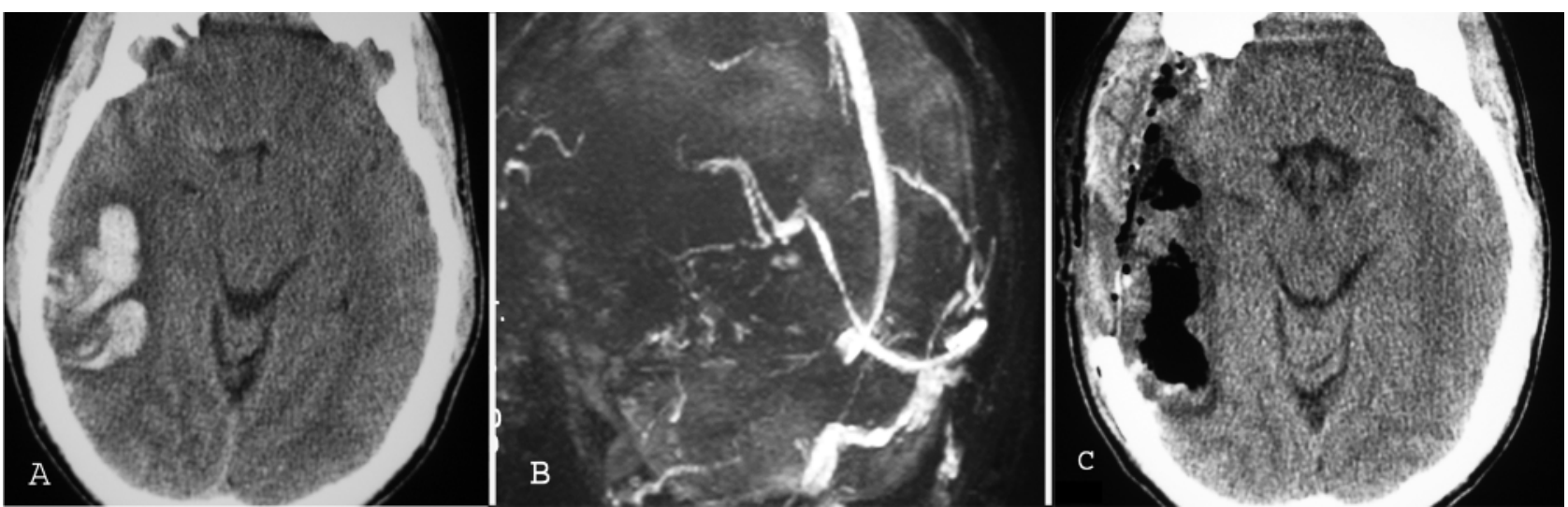

FIG. 3. Case 3. A: Preoperative CT revealing a hemorrhagic infarction of the right temporal lobe with transtentorial herniation. B: An MR venogram showing absence of flow in the transverse sinus and Labbe vein on the right side. C: Postoperative CT of the brain. 
Craniectomy and dural sinus thrombosis

TABLE 1: Summary of published clinical series and case-reports on decompression for CVDST*

\begin{tabular}{|c|c|c|c|c|c|}
\hline Authors \& Year & $\begin{array}{l}\text { GCS } \\
\text { Score }\end{array}$ & Pupillary Signs & Dural Sinus & Decompression & $\begin{array}{l}\text { Outcome } \\
\text { (GOS) }\end{array}$ \\
\hline \multirow[t]{3}{*}{ Stefini et al., 1999} & 4 & bilaterally dilated & transverse \& sigmoid & ED & GR \\
\hline & 6 & bilaterally dilated & transverse \& sigmoid & ED & GR \\
\hline & 5 & bilaterally dilated & transverse \& sigmoid & ED \& drainage of a superficial clot & SD \\
\hline Weber \& Spring, 2004 & $<8$ & unilaterally dilated & transverse \& sigmoid & ED & MD \\
\hline \multirow[t]{4}{*}{ Keller et al., 2005} & 9 & normal & superior sagittal & ED \& ID & GR \\
\hline & 6 & normal & frontal cortical veins & $E D \& I D$ & GR \\
\hline & 13 & normal & transverse \& sigmoid & $E D \& I D$ & MD \\
\hline & 13 & normal & transverse \& sigmoid & ED & MD \\
\hline \multirow[t]{3}{*}{ present series } & 3 & bilaterally dilated & transverse \& sigmoid & $E D \& I D$ & MD \\
\hline & 5 & unilaterally dilated & superior sagittal & \& & GR \\
\hline & 8 & unilaterally dilated & transverse \& sigmoid & ED and ID & GR \\
\hline
\end{tabular}

* Based on MEDLINE and EMBASE search from 1970 to 2008. Abbreviations: ED = external decompression (defined as craniectomy and duraplasty); GCS = Glasgow Coma Scale; GOS = Glasgow Outcome Scale; GR = Good Recovery; MD = Moderate Disability; ID = internal decompression (defined as hematoma drainage and resection of necrotic tissue); SD = Severe Disability.

Outcome. After 1 month the patient's GOS score indicated good recovery. He did not suffer from seizures. The neuropsychological assessment was normal.

\section{Discussion}

The clinical history of these 3 patients supports the hypothesis that emergency DS may be life-saving in patients with transtentorial herniation due to CVDST and that both the functional and the neuropsychological outcome may be favorable, even in deeply comatose patients with ominous pupillary signs. No intracranial bleeding complications directly related to the DS occurred and no patient developed postoperative hydrocephalus.

Consistent with the rarity of the pathology, a MEDLINE and EMBASE search of the literature from January 1970 to October 2008 using 6 key words (cerebral, dural sinus, vein, thrombosis, surgery, decompression) revealed only 3 surgical reports on patients (a total of 8) who underwent DS for CVDST. ${ }^{11,17,20}$ Four of these 8 patients had pupillary signs of brain herniation ${ }^{17,20}$ (Table 1).

Our unexpectedly favorable results were consistent with those reported in the literature. Including our 3 patients, 6 of the 7 patients who were moribund with pupillary signs were independent in daily living after discharge from the rehabilitation units. ${ }^{17,20}$ Furthermore, Keller and colleagues ${ }^{11}$ observed a good functional outcome (moderate disability, 2 cases; good recovery, 2 cases) in all 4 of their patients who underwent DS before developing clinical signs of herniation (Table 1). The 2 patients who were categorized as having moderate disability suffered from slight focal signs probably related to the eloquence of the cortex involved in the hemorrhagic infarction. ${ }^{11}$

Although the analysis of case reports and small case series may be affected by publication bias and a formal statistical investigation of confounding variables and procedure-related risk factors is impossible, the reported clinical results are worthy of attention for at least 2 reasons: 1) according to the ISCVT analyses, all patients had one or more of the main independent predictors of death (intracranial hemorrhage, clinical worsening, GCS score $<9$, new focal sign); ${ }^{7}$ and 2) the most frequent CVDST mechanism of death is transtentorial herniation, and 7 of the 11 patients already had related clinical signs. ${ }^{2}$

Explanations of how DS may affect the physiopathology of CVDST can only be speculative. Cerebral venous and dural sinus thrombosis causes a wide spectrum of parenchymal changes, ranging from vasogenic and cytotoxic edema to intracerebral hematomas.,5 These complications may lead to a compartmentalized intracranial hypertension that secondarily compresses intraparenchymal veins, with a subsequent worsening of edema and swelling. Furthermore, in CVDST the regional cerebral blood flow may be reduced because of paradoxical arteriolar vasoconstriction. ${ }^{5,19}$ A condition that, based on experimental data, has features reminiscent of ischemic penumbra. ${ }^{18}$ Decompressive surgery creates space for the swelling brain and, by reducing intracranial hypertension and brainstem compression, may break the vicious cycle by relieving arteriolar constriction and restoring flow in compressed venous collaterals.

In view of the rarity of patients with malignant CVDSTs necessitating emergency DS, the surgical experience is limited and accepted guidelines on how to perform the decompression are lacking. The review shows that there is no unanimity as to what constitutes the preferable surgical strategy. Different authors have adopted distinct approaches: pure external decompression (in 5 cases) or internal decompression with or without removal of the bone flap (in 6 cases) (Table 1). A statistical comparison of the results obtained with the 2 strategies is beyond the limits of small series because of limited power and clinical, timing, and risk-factor heterogeneity among the studies.

In our experience, we tried to define criteria a priori to guide surgical decision making. In particular, we considered the site and characteristics of the clot and the eloquence of the brain, with the aim of tailoring surgery and 
reducing its burden in a situation where cytotoxic edema may coexist with viable brain. ${ }^{5,13}$ In practice, the peculiarities of the cerebral lesions (for example, site, clot dimension, depth), together with the clinical course and the supposed hemispheric dominance, guided our decisions toward internal decompression with removal of the bone flap in all cases. In the light of the favorable neuropsychological outcomes, this experience appears reasonable and is in accordance with that of other authors. ${ }^{11}$

On the other hand, other case reports have shown almost equally favorable results with pure external decompressions. ${ }^{17,20}$ Recently, MR imaging studies have demonstrated the existence of parenchymal changes (vasogenic edema) that could be completely reversible, and such studies may be advocated in support of a less invasive surgical approach. ${ }^{16}$ From the analysis of the cases in which patients were treated by means of external decompression, the importance of surgical timing emerges. Stefini et al. ${ }^{17}$ observed that the ICP can be better controlled when the operation is promptly performed. The importance of timing may be indirectly supported by the recent trials investigating external decompression for patients with space-occupying arterial infarcts. ${ }^{10}$

In conclusion, although the rarity of the condition precludes any statistically based evidence, the functional recovery obtained in a situation in which the prognosis would otherwise have been grim ${ }^{15}$ appears clinically relevant. Decompressive surgery may offer the last chance of life and a favorable functional outcome in selected patients with CVDST whose condition is deteriorating because of impending brain herniation that is unresponsive to causal therapy and intensive care. In the future, updates on the pathophysiology of CVDST and new developments in imaging technologies will provide a better and more comprehensive insight into the specific therapeutic requirements of each patient. A patient-specific pathophysiologically based approach could enable the clinician to choose the appropriate surgical strategy and timing with more accuracy and, probably, greater effectiveness.

\section{Disclosure}

The authors report no conflict of interest concerning the material or methods used in this study or the findings specified in this paper.

\section{Acknowledgments}

The authors thank Mario Lanterna and Alida Scolari for their invaluable advice.

\section{References}

1. Bousser M-G, Ferro JM: Cerebral venous thrombosis: an update. Lancet Neurol 6:162-170, 2007

2. Canhão P, Ferro JM, Lindgren AG, Bousser MG, Stam J, Barinagarrementeria F; ISCVT Investigators: Causes and predictors of death in cerebral venous thrombosis. Stroke 36:1720 1725,2005

3. Chu K, Kang DW, Yoon BW, Roh JK: Diffusion-weighted magnetic resonance in cerebral venous thrombosis. Arch Neurol 58:1569-1576, 2001

4. de Bruijn SFTM, Stam J : Randomized, placebo-controlled trial of anticoagulant treatment with low-molecular-weight heparin for cerebral sinus thrombosis. Stroke 30:484-488, 1999
5. Doege CA, Tavakolian R, Kerskens CM, Romero BI, Lehmann R, Einhäupl KM, et al: Perfusion and diffusion magnetic resonance imaging in human cerebral venous thrombosis. J Neurol 248:564-571, 2001

6. Einhäupl K, Bousser MG, de Bruijn SFTM, Ferro JM, Martinelli I, Masuhr F, et al: EFNS guideline on the treatment of cerebral venous and sinus thrombosis. Eur J Neurol 13:553559,2006

7. Ferro JM, Canhao P, Stam J, Bousser M-G, Barinagarrementeria F; ISCVT Investigators: Prognosis of cerebral vein and dural sinus thrombosis. Results of the International Study on Cerebral Vein and Dural Sinus Thrombosis (ISCVT). Stroke 35:664-670, 2004

8. Gerlach R, Scheuer T, Beck J, Woszczyk A, Böhm M, Seifert $\mathrm{V}$, et al: Risk of postoperative hemorrhage after intracranial surgery after early Nadroparin administration: results of a prospective study. Neurosurgery 53:1028-1035, 2003

9. Girot M, Ferro JM, Canhao P, Stam J, Bousser M-G, Barinagarrementeria F, et al : Predictors of outcome in patients with cerebral venous thrombosis and intracerebral hemorrhage. Stroke 38:337-342, 2007

10. Hofmeijer J, Kappelle LJ, Algra A, Amelink GJ, van Gijn J: Bart van der Worp H, for the HAMLET investigators. Lancet Neurol 8:326-333, 2009

11. Keller E, Pangalu A, Fandino J, Konu D, Yonekawa Y: Decompressive craniectomy in severe cerebral venous and dural sinus thrombosis. Acta Neurochir Suppl (Wien) 94:177-183, 2005

12. Lasjaunias P, Berenstein A, Ter Brugge KG: Intracranial venous system, in Lasjaunias P, Berenstein A, Ter Brugge KG (eds): Surgical Neuroangiography, ed 2. Berlin, Heidelberg, New York: Springer-Verlag, 2001, Vol 1, pp 675-678

13. Leach JL, Fortuna RB, Jones BV, Gaskill-Shipley MF: Imaging of cerebral venous thrombosis: current techniques, spectrum of findings, and diagnostic pitfalls. Radiographics 26 (1 Suppl):S19-S41, 2006

14. Lensing AWA, Prins MH, Davidson BL, Hirsh J: Treatment of deep venous thrombosis with low-molecular-weight heparins: a meta-analysis. Arch Intern Med 155:601-607, 1995

15. Petzold A, Smith M: High intracranial pressure, brain herniation and death in cerebral venous thrombosis. Stroke 37:331332, 2006 (Letter)

16. Röttger C, Trittmacher S, Gerriets T, Blaes F, Kaps M, Stolz E: Reversible MR imaging abnormalities following cerebral venous thrombosis. AJNR Am J Neuroradiol 26:607-613, 2005

17. Stefini R, Latronico N, Cornali C, Rasulo F, Bollati A: Emergent decompressive craniectomy in patients with fixed and dilated pupils due to cerebral venous and dural sinus thrombosis: a report of three cases. Neurosurgery 45:626-629, 1999

18. Ungersbock K, Heimann A, Kempski O: Cerebral blood flow alterations in a rat model of cerebral sinus thrombosis. Stroke 24:563-569, 1993

19. Villringer A, Mehraein S, Einhäupl KM: Pathophysiological aspects of cerebral sinus venous thrombosis (SVT). J Neuroradiol 21:72-80, 1994

20. Weber J, Spring A: [Unilateral decompressive craniectomy in left transverse and sigmoid sinus thrombosis.] Zentralbl Neurochir 65:135-140, 2004 (Ger)

Manuscript submitted January 10, 2009.

Accepted March 30, 2009.

Portions of this work were presented in poster form at the 57th Italian Conference of Neurosurgery, Udine, Italy, November 8, 2008.

Address correspondence to: Luigi A. Lanterna, M.D., Ph.D., Via B Crespi, 13 Milano, Italy. email: 1.lanterna@virgilio.it. 